\title{
OPTIMIZATION OF ARQ PARAMETERS OF STANAG 5066 FOR THE HFDVL MODEM
}

\author{
I. Raos*, S. Zazo-Bello*, I. Pérez-Álvarez ${ }^{\dagger}$,J. López-Pérez ${ }^{\dagger}$ L. Melián-Gutiérrez ${ }^{\dagger}$ B. Pérez-Díaz ${ }^{\dagger}$ \\ *Universidad Politécnica de Madrid (UPM), Dpto. SSR, E.T.S. Ingenieros de Telecomunicación, Ciudad Universitaria, 28040 \\ Madrid, Spain, e-mail: ivana@gaps.ssr.upm.es \\ †Instituto para el Desarrollo Tecnológico y la Innovación en Comunicaciones (IDeTIC), Universidad de las Palmas de G. C. \\ Campus de Tafira, 35017 Las Palmas G.C. Spain, e-mail: iperez@idetic.eu
}

Keywords: HF modem, SRQ, STANAG 5066, data rate, delay, optimization

\begin{abstract}
The reliability of bidirectional communication link can be guaranteed with Automatic Repeat Request Procedures (ARQ). The standard STANAG 5066 describes the ARQ procedure for HF communications that can either be applied to existing HF physical layers modems or adapted to future physical layer designs. In this contribution the physical layer parameters of an HF modem (HFDVL), developed by the authors over the last decade, are chosen to optimize the performance of the ARQ procedure described in STANAG 5066. Besides the interleaving length, constellation size and coding type, the OFDM-based HFDVL modem permits the selection of the number of receiver antennas. It will be shown that this parameter gives additional degrees of freedom and permits reliable communication over low SNR HF communication links.
\end{abstract}

\section{Introduction}

HFDVL modem is an HF modem that reaches data rates up to $8640 \mathrm{~b} / \mathrm{s}$ in the $3 \mathrm{KHz}$ bandwidth, designed and developed by the authors and described in several publications (see for instance [1]). The performances of the modem have been improving over the last years, especially with the addition of multiple antennas at receiver, known as Single Input Multiple Output (SIMO) system [2].

At this point, we have already shown very satisfactory behaviour in broadcast mode with high reliability. Current goal is to add reliability using ARQ procedures in point-topoint mode (bidirectional communication link). Nevertheless, it must be noted that there is a number of applications for which large transmission delay is unaffordable, and ARQ procedures cannot be applied (digital voice transmission, for instance).

The analysed point-to-point scenario includes an ARQ procedure whose parameters should be chosen according to the physical layer modem characteristics. In this contribution, the simplified ARQ procedure from standard STANAG 5066 for normal data transfer ([3]), Selective Repeat Request (SRQ) is analysed with the HFDVL modem as the physical layer. The selection of the STANAG 5066 as a framework is due to its current importance that probably will increase in the forthcoming years.

The aim is to find the set of optimal SRQ parameters for HFDVL modem when receiver processes either single or multiple antennas outputs (SISO and SIMO systems). The optimality is measured in terms of information data rate with SRQ (including retransmissions and overhead), as well as the introduced delay. In order to perform these analyses, both theoretical and real transmission results will be reported.

In the first part of this contribution, an introduction to the HFDVL modem is given, followed by the short description of SRQ STANAG 5066 procedure and explanation of the adaptation of SRQ parameters to HFDVL modem. The simulations are performed to confirm that the known formulas can be used for the estimation of the number of retransmissions with HFDVL modem. In the next section, the maximum achievable data rate and minimum delay are obtained as the system bounds and simulation in moderate HF channel (the real communication links between Madrid and Las Palmas de Gran Canaria has this statistics) with one chosen configuration of the whole system are performed to obtain FER performances. The transmission of one file is simulated with SRQ procedure to estimate the loss with respect to the maximum achievable performances (in terms of data rate and delay). Finally, real transmission results, with simplified ARQ, are used to verify the theoretical studies.

\section{HFDVL modem physical layer basics}

HFDVL modem is an OFDM-based modem for data and digital voice transmission. It has three different sources of diversity: time diversity, achieved by interleaving; frequency diversity, as it is flexible to change the frequency range of communication if performances are not acceptable, and spatial diversity as multiple receiver antennas signals can be combined.

In this contribution, the priority is given to the analysis of spatial diversity, and receivers with 1, 2 and 4 antennas are analysed ([4]). The source of time diversity, that is the interleaving size, is maintained fixed. The long interleaving, that is 5seconds long, it results in largest time diversity and is considered for the parameter optimization. The influence of 
the frequency diversity is not analysed either, as all transmissions are performed over a single frequency band.

The HFDVL modem modes are resumed in Table 1 . The parameters that can be changed are constellation (QPSK, 16QAM or 64QAM), coding (1/2 and 4/5 LDPC codes) and interleaving (short, medium and long whose duration are $1.67,3.33$ and $5 \mathrm{~s}$, respectively). The election of interleaving length does not influence the data rate, but only the system performances (larger length enables higher level of time diversity and better error protection).

\begin{tabular}{|c|c|c|c|c|c|c|}
\hline constellation & \multicolumn{2}{|c|}{ QPSK } & \multicolumn{2}{c|}{ 16QAM } & \multicolumn{2}{c|}{ 64QAM } \\
\hline coding & $1 / 2$ & $4 / 5$ & $1 / 2$ & $4 / 5$ & $1 / 2$ & $4 / 5$ \\
\hline data rate $[\mathrm{kb} / \mathrm{s}]$ & 1.8 & 2.88 & 3.6 & 5.76 & 5.4 & 8.64 \\
\hline
\end{tabular}

Table 1 HFDVL modem transmission modes

Out of all available modes, the one with 16QAM constellation and $1 / 2$ LDPC code is chosen. This mode results in $3.6 \mathrm{~kb} / \mathrm{s}$ data rate. The ALE modem sets this configuration and it is maintained during the transmission. We assume that the channel does not change its statistics during the transmission, although its time variability is simulated. The computer simulations are performed using Wattersson HF channel model that is CCIR compliant.

\section{SRQ procedure}

\subsection{STANAG 5066 Sliding Window}

The SRQ algorithm consists in the encapsulation of information data (its size is not fixed by standard 5066, can be up to 1023 bytes long) in frames denoted D-PDU. The added overhead consists of two CRC codes defined in [5] (for header and information data), synchronization sequence and some management information bits.

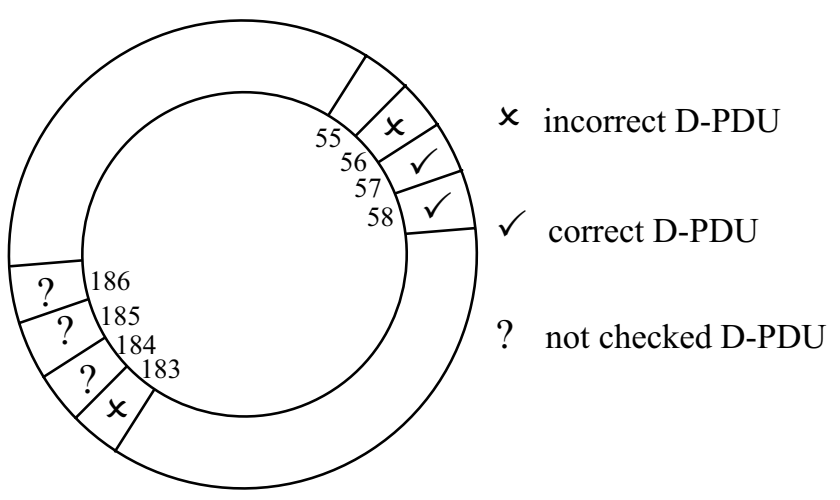

Figure 1 Simplified circular sliding window buffer scheme

The mere SRQ procedure consists in the transmission of a number of D-PDU (up to 128 in standard 5066) followed by the ACK in which correct or incorrect reception of D-PDU is confirmed. The simplified sliding window circular buffer is shown in Figure 1. The sliding window size determines the maximum available memory for not-acknowledged D-PDU. If it is 128 (the maximum permitted value), and, for example, the first not-acknowledged D-PDU is in buffer position 56, the last received D-PDU that is processed at receiver (it is checked if it is correctly received) is stored in position $56+128-1=183$. In the example in Figure 1, that PDU is not correctly received. The next received D-PDU is stored in buffer (at most 128 additional D-PDU can be stored), but its correct reception is not checked (that is the reason for the question mark in positions $184,185,186)$. When D-PDU stored in position 56 is received correctly, the start point of the sliding window will move to the next incorrectly received D-PDU (in this example, as D-PDU at positions 57 and 58 are correctly received, it will advance at least 3 positions).

\subsection{An example of HFDVL modem adaptation}

In this example, the communication scenario is chosen to be the one whose maximum continuous transmission time is as close as possible to the maximum continuous transmission interval permitted by STANAG 5066 (127.5s). The number of D-PDU equals the maximum sliding window size (128).

The mapping between D-PDU and OFDM symbols is shown schematically in Figure 2 where $N$ D-PDU with zero padding are mapped into $M$ interleaving units.

The basic block of HFDVL modem is one interleaving unit. With long interleaving of $5 \mathrm{sec}$, 16QAM, and $1 / 2$ LDPC coding rate, it contains a total of 18000 bits. Each interleaving unit (with long interleaving) contains 150 OFDM symbols.

In maximum continuous time there can be a total of 25 basic blocks. The total frame duration, $125.2971 \mathrm{~s}$, is obtained adding the header and OFDM symbol, indicator of end of transmission.

The total number of bits in continuous transmission interval is 450000 bits, and there should be encapsulated 128 D-PDU. This gives the D-PDU size of 3515 bits, out of which 128 are control and synchronization bits. Therefore, HFDVL modem will be configured to have 3387 info bits in one D-PDU.

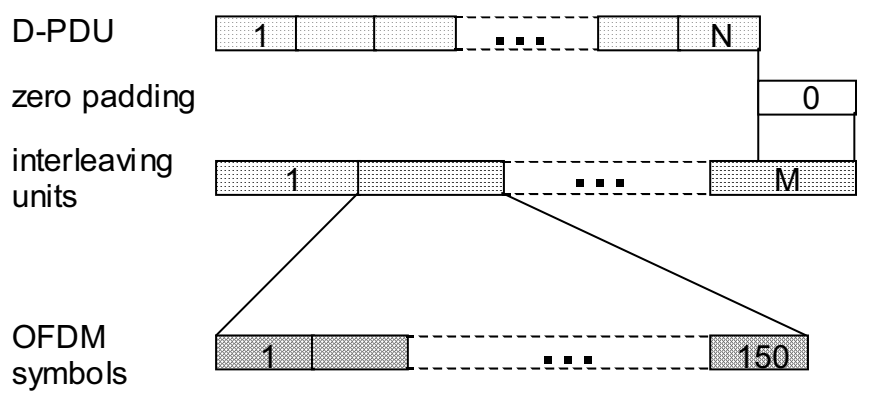

Figure 2 Mapping of D-PDU in OFDM symbols

\subsection{Sliding Window in HF channel}

Assuming error-free transmissions of ACK and NAK frames, and independent errors in different D-PDU, the probability of requiring exactly $k$ attempts to successfully receive a given frame is

$$
q_{k}=(1-F E R) \times F E R^{k-1}, k=1,2, \ldots
$$

where FER is D-PDU error probability.

In order to corroborate that the independency assumption between D-PDU can be applied in HFDVL modem communications with chosen parameters, the scenario with $\mathrm{SNR}=7 \mathrm{~dB}$ and single receiver antenna is simulated, for PDU 
info size of 434 bits (a transmission frame consists of 4 interleaving units), in moderate CCIR channel. The results in two independent channel realizations are shown in Figure 3. On $x$-axis is the number of transmissions until correct D-PDU reception and on $y$-axis is shown the probability of having given number of transmissions. Both theoretical and simulated results are shown. It can be observed that the simulated results are very close to the ones described by (1), and that this formula can be used for delay estimation.
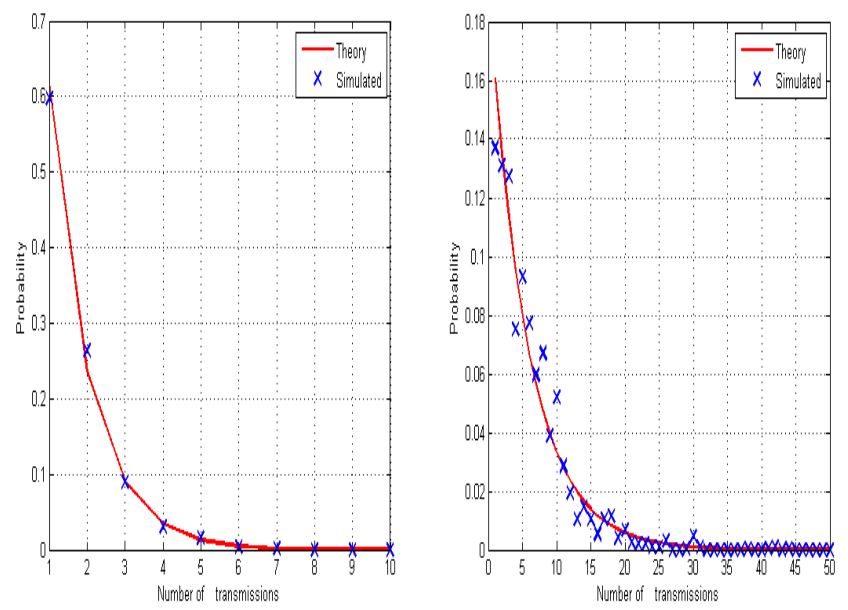

Figure 3 Probability of having the given number of transmissions for FER $=0.38$ (left) and 0.84 (right)

The average delay corresponding to FER lower than 1 is found according to the average number of retransmissions $\left(N_{\text {av }}\right)$ needed for a D-PDU to be successfully accepted by the receiver, being $p$ the probability of successful D-PDU transmission [6]:

$$
N_{a v}=p+2 p(1-p)+3 p(1-p)^{2}+\ldots=\frac{1}{p}=\frac{1}{1-F E R}
$$

The goodness of this formula in HF moderate channel is confirmed by simulation of SRQ procedure with uniformly distributed errors and with HFDVL modem with information D-PDU size of 434 bits assuming fixed transmission frame size of 4 interleaving units. The results in Figure 4 show that simulated performances fit the theoretical.

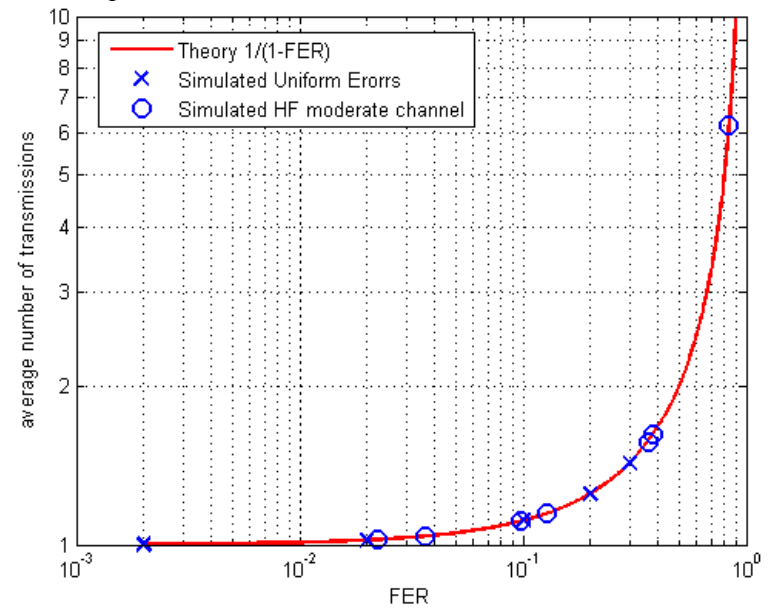

Figure 4. Average number of transmissions for a given FER

\section{Parameters optimization}

The theoretical studies consider D-PDU overhead bits as defined in STANAG, a total of 16 byes (including header and info CRC bits, synchronization and management data). In this study we assume error-free reception of ACK frames to simplify the simulation task. In the delay calculation, the propagation time and ACK frames duration was not taken into account, considering it much lower than transmission frame duration.

\subsection{System bounds}

The maximum achievable data rates and corresponding minimum delays (under $F E R=0$ condition) is shown in Figure 5. With larger (realistic) FER the delay will be larger and data rate will be lower, and working points will be situated inside the green area shown Figure 5. Each point (marked with cross) corresponds to different transmission frame length from 1 to 25 interleaving units. It can be observed that the highest data rate can be achieved when transmission frame consists of 25 interleaving units, although it also results in maximum delay.

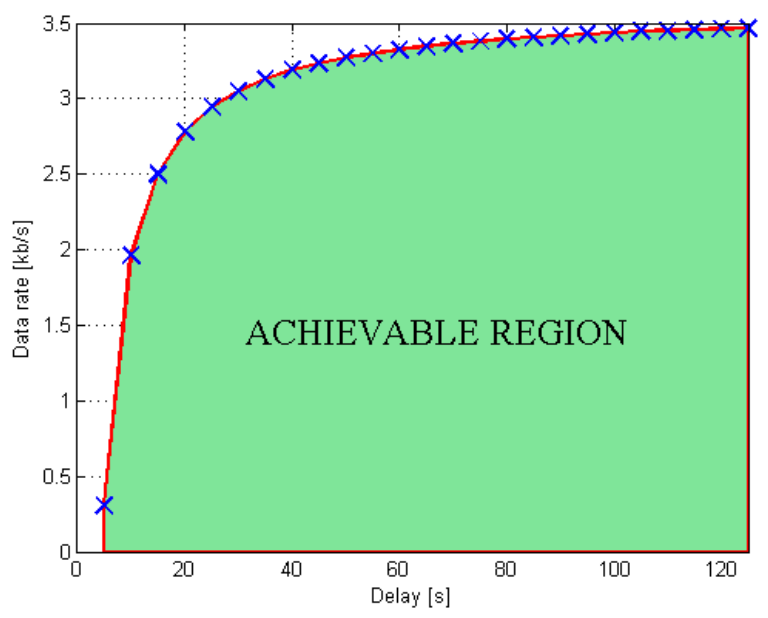

Figure 5 Theoretical system bounds

\subsection{Simulated scenario}

HFDVL modem with D-PDU size of 3387 information bits and maximum transmission frame of $128 \mathrm{D}-\mathrm{PDU}$ is analysed in terms of delay and data rate. Simulations are performed in at least 500 channels realizations of HF moderate channel with 1,2 and 4 receiver antennas.

The first analysis considers the number of channels in which communication is not possible, as FER equals 1 . These results are shown in Figure 6 where percentage of channel realizations with FER equal to 1 is shown.

It can be observed that with single, two and four receiver antennas the limit SNR values with less than $5 \%$ of channels in which no communication is possible are 8,6 and $6 \mathrm{~dB}$, respectively. Therefore, if channel SNR is higher that these bounds, it can be guaranteed that at least in $95 \%$ of channel realization the communication will be possible (FER will be below 1). 


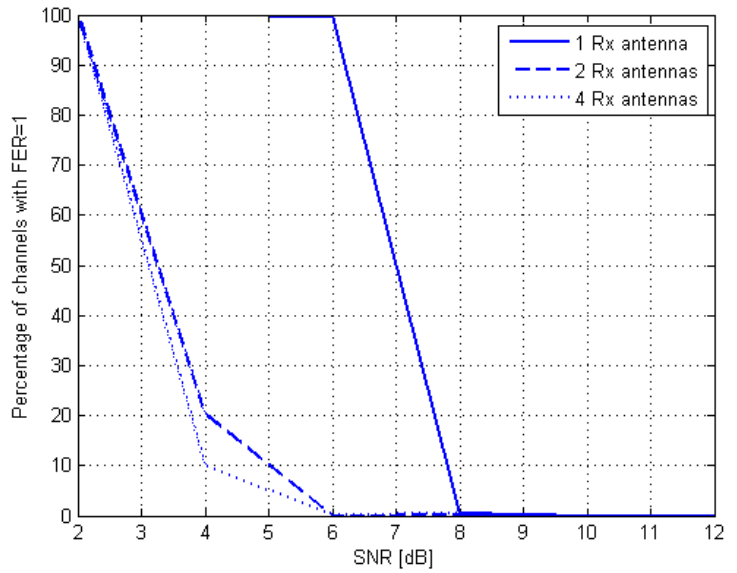

Figure 6 Statistics of FER=1

The FER distribution is shown in Figure 7. Comparing results at $\mathrm{SNR}=4 \mathrm{~dB}$, with 2 and 4 receiver antennas, it can be observed that the FER with 2 receiver antennas is very concentrated between FER values of 0.9 and 1, while for 4 receiver antennas FER values as low as 0.5 can be achieved, and in more than $75 \%$ of channels FER is below 0.9 . It can be seen that if FER below 0.1 is aimed, the SNR levels at the channel should be at least 12,8 and 6 for 1,2 and 4 receiver antennas, respectively.

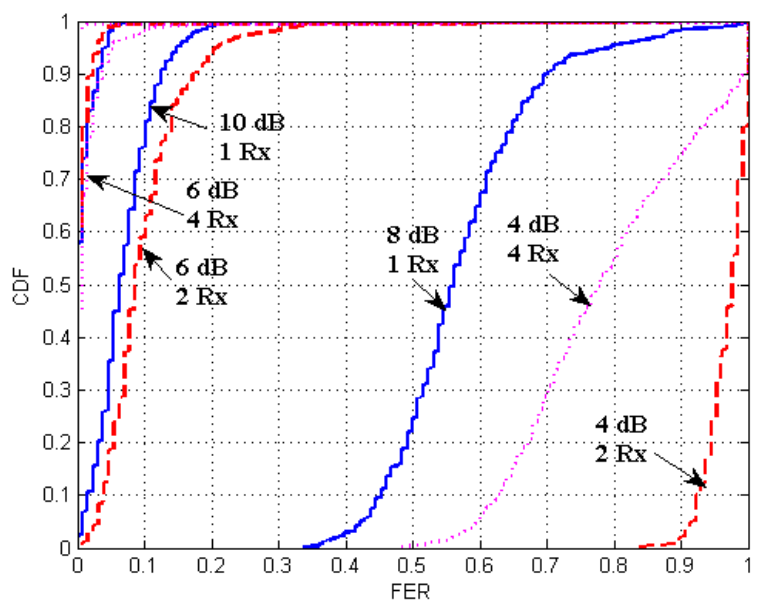

Figure 7 FER distributions of simulated systems

\subsection{File transmission}

The transmission of 500kbytes file is simulated with SRQ algorithm and info D-PDU size of 3387 bits. The sliding window size is set to 128 , and the memory to 256 D-PDU. The maximum continuous transmission interval contains 128 PDU. Under these assumptions, in transmission without DPDU errors, the data would be transmitted in 10 frames with 128 D-PDU each, resulting in data rate of $3200 \mathrm{~b} / \mathrm{s}$ and $1250 \mathrm{~s}$ delay. This represents the bounds for given file transmission. The highest theoretical data rate in this configuration, $3468.3 \mathrm{~b} / \mathrm{s}$, cannot be achieved as this bound corresponds to transmission of frames with 128 D-PDU (the best efficiency) and list of infinite bits to be transmitted.

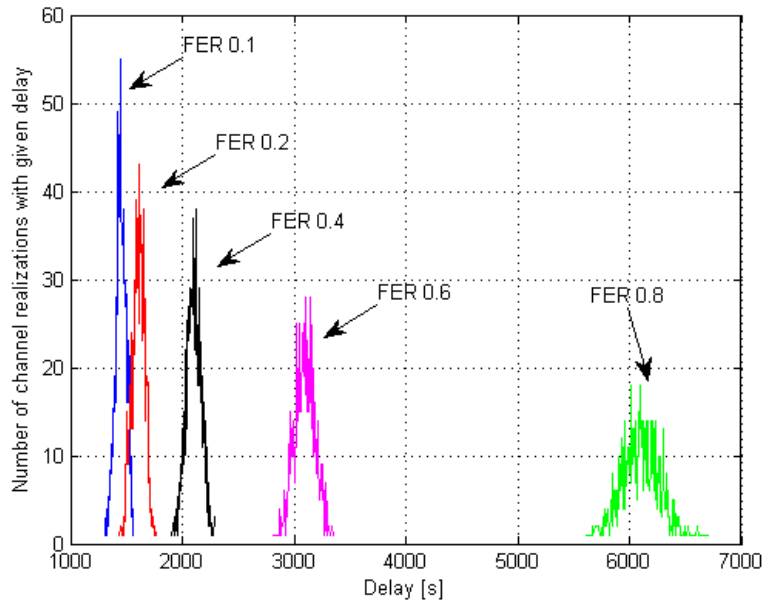

Figure 8 Delay distributions for different FER values

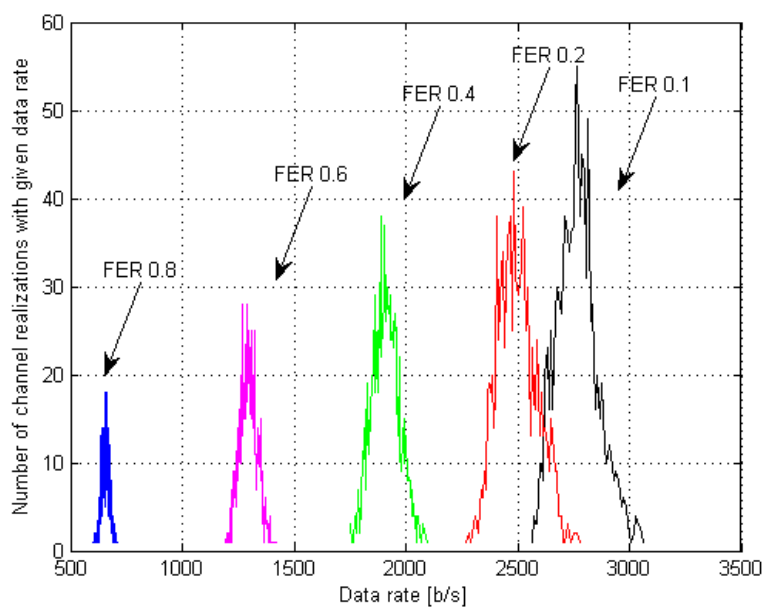

Figure 9 Data rate distributions for different FER values

The simulations are performed for different FER values and delay and data rate results are shown in Figure 8 and Figure 9. It can be observed how delay becomes more concentrated for lower FER values. The values for FER 0.01 are not represented graphically as they present very big peak, as in $88.6 \%$ of channels realizations the data rate of $2909.1 \mathrm{~b} / \mathrm{s}$ with $1375 \mathrm{~s}$ delay is achieved. In the actual transmission, the highest data rate was $3149.6 / \mathrm{s}$ with $1270 \mathrm{~s}$ delay and is reached for FER 0.01 .

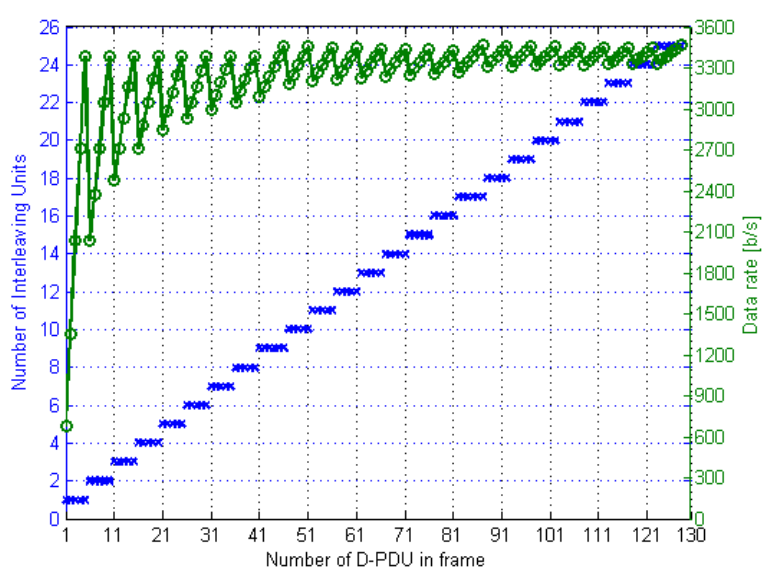

Figure 10 System statistics for different D-PDU lengths 
For transmission of finite file sizes, it is interesting to observe in the Figure 10 the achievable data rate and the frame size (in number of interleaving units) depending on the number of available data (number of D-PDU that are available for transmission).It can be observed that whenever the number of D-PDU is multiple of 5, the data rate is close to the upper data rate bound. This is due to the ratio between total number of bits in 5 D-PDU and 1 interleaving unit (17 575 and 18 000).

\section{Real transmission results}

The real system operates at different SNR that can be estimated at receiver. Incorporation of multiple antennas at receiver permits signal detection at lower SNR and permits better analysis of real channel SNR values. Currently, the available results are not SNR classified, but represent the averaged data rates of a real link between Madrid and Gran Canaria. The simplified ARQ scenario considers error-free ACK messages and no control data are added to D-PDU (no loss of data rate due to signalling is taken into account).

In Figure 11 is shown the distributions of data rates of all the configurations of the HFDVL (with 2 receiving antennas) in comparison with the STANAG $4285 \mathrm{C}$ in a real link. Three different D-PDU sizes were considered: 100, 500 and 1000 bits. The higher D-PDU size enables higher efficiency, but results in higher number of erroneous D-PDU than shorter DPDU would. That is the reason for wider range of data rate with higher D-PDU size. Comparing these real system results with simulated for D-PDU 3387 size, it can be observed that the SNR in the HF channel corresponds to FER of 0.1 or lower. Although ideal in some extent, these results corroborate that a suitable ARQ will guarantee a very significant improvement in front of standard alternatives.

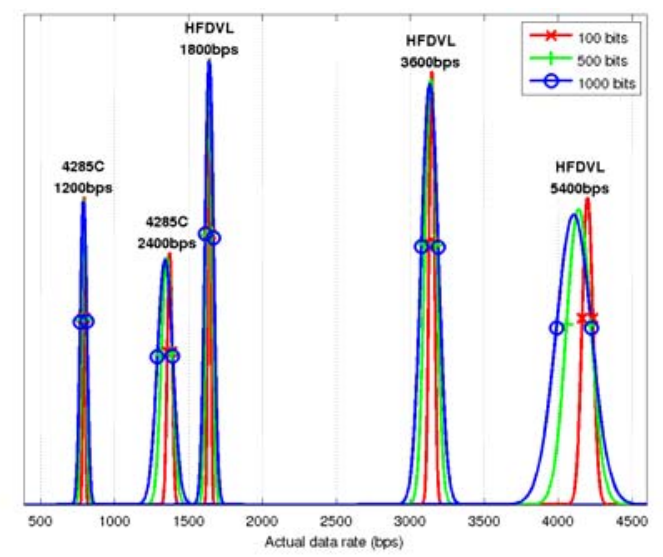

Figure 11 Real system data rate

\section{Conclusions}

In this contribution the adaptation of HFDVL modem to STANAG 5066 SRQ protocol was studied.

Different D-PDU sizes and their efficiency is analysed in terms of latency and data rate. Lower D-PDU sizes lead to lower data rate but also to lower delays. Therefore, the choice of the number of information bits that can form one D-PDU unit depends both on the channel imposed restriction in terms of FER and the application and its requirements in terms of delay and objective data rate.

It is shown that addition of multiple antennas in receiver improves performances both in terms of delay and data rate in up to $4 \mathrm{~dB}$ in SNR. It should be also noted that the gain of 2 antennas system with respect to single antenna system is greater than the gain of 4 antenna system with respect to 2 antenna system. Therefore, multiple antennas enable higher data rates and lower delay systems.

The finite length file transmission was simulated with SRQ algorithm and it showed that FER of the order of 0.01 a mean data rate is $10 \%$ away from the maximum achievable rate with simulated configuration $(2909 \mathrm{~b} / \mathrm{s}$ vs $3200 \mathrm{~b} / \mathrm{s})$. The maximum achievable rate with $500 \mathrm{kbytes}$ file is $11 \%$ lower than the HFDVL modem limit reached without ARQ procedure implementation $(3200 \mathrm{~b} / \mathrm{s}$ vs $3600 \mathrm{~b} / \mathrm{s})$, The transmission of the infinite list of bits would result in $3.66 \%$ loss $(3468.3 \mathrm{~b} / \mathrm{s}$ vs $3600 \mathrm{~b} / \mathrm{s})$.

The simplified real transmission results showed the data rate loss of $9 \%$, without considering the data rate loss due to signalling overhead that would add an additional 3.66\% loss for configuration chosen for simulations.

\section{Acknowledgements}

This work was supported by the Spanish Ministry of Science and Innovation under grants TEC2010-21217-C02 CR4HFDVL, and CONSOLIDER-INGENIO 2010 CSD2008-00010 COMONSENS.

\section{References}

[1]: Santana, H.; Zazo, S.; Pérez, I.; Raos, I.; Mendieta, E.; López, J. Validation of a HF Spread Spectrum MultiCarrier Technology through real Link Measurements. European Trans. on Telecomunications.Vol.:17, Nov-Dec 2006

[2] I. Pérez-Álvarez, S. Zazo Bello, M. Ghogho, J. López-Pérez "Experimental Results on Multicarrier MIMO HF Communications", General Assembly and Scientific Symposium of the International Union of Radio Science. Istanbul, Turkey. August $13-20,2011$

[3] STANAG 5066: Profile for HF Data Communications V2, Publication Date: Dec 5, 2008

[4]: Peco, C.; Zazo-Bello, S.; Pérez-Álvarez, I.; López-Pérez, J.On the diversity in multiantenna HF communications, IRST 2009: 11th International Conference on Ionospheric Radio Systems \& Techniques. Edinburgh, U.K.; April 2009

[5] J. K. Wolf and R. D. Blakeney II, "An exact evaluation of the probability of undetected error for certain shortened binary CRC codes", in IEEE Conference on Military Comm., MILCOM 1988, pp 287-292, paper 15.2

[6] S. Lin, D. J. Costello, Jr, "Error Control Coding, Fundamentals and Applications", Second Edition 2004, ISBN $0-13-042672-5$ 